\title{
TF-CBT Informed Teletherapy for Children with Autism and their Families
}

\author{
Justin S. Romney ${ }^{1}$ (D) Miranda Garcia ${ }^{1}$ \\ Accepted: 13 April 2021 / Published online: 20 April 2021 \\ (C) The Author(s), under exclusive licence to Springer Nature Switzerland AG 2021
}

\begin{abstract}
A diagnosis of autism spectrum disorder (ASD) often puts a child in a vulnerable position. While the research of the effects of trauma on children diagnosed with ASD is limited, we know children diagnosed with ASD presenting with a comorbid diagnosis of post-traumatic stress disorder (PTSD) have an increased risk of suicidal thoughts and behaviors. TF-CBT is an empirically validated treatment for trauma; in this paper, we provide adaptions for using this treatment with children diagnosed with ASD and using this method for teletherapy. These adaptions include recognizing trauma behaviors and ASD behaviors, the use of repetition and the need for flexibility from the therapist, and addressing safety with ASD behaviors while working from a teletherapy platform.
\end{abstract}

Keywords Trauma $\cdot$ Autism Spectrum disorder $\cdot$ TF-CBT $\cdot$ Teletherapy

Trauma comes from an event or series of events that are threatening to the individual and impact immediate and long-term functioning (Kerns et al., 2015). No one person experiences traumatic events the same; two individuals could experience the same event with differing perceptions and reactions. One could experience PTSD-like symptoms, while another does not react (Peterson et al., 2019). Trauma is a psychological wound that has long-lasting effects on the functioning of the individual. The severity, length, and permanency of the trauma vary widely due to the diversity of psychological, social, and physical functioning (Kerns et al., 2015).

Children diagnosed with autism spectrum disorder (ASD) presenting with a comorbid diagnosis of post-traumatic stress disorder (PTSD) have an increased risk of suicidal thoughts and behaviors (Storch et al., 2013). Research has shown that up to $11 \%$ of children with this combination of diagnoses have intrusive suicidal thoughts (Storch et al., 2013). The research on the effects of trauma on individuals diagnosed with ASD is limited (Brenner et al., 2017); we know that these children are at increased risk of being victims of trauma (Peterson et al.,

Justin S. Romney

justin.s.romney@ttu.edu

1 Department of Community, Family, and Addiction Sciences, Texas Tech University, 1301 Akron Ave., MS 41250,

Lubbock, TX 79409-1250, USA
2019). With the prevalence of ASD continually increasing, it is more likely that these children and their families will be seeking therapy.

The Covid-19 pandemic has highlighted the need for direction on how to use teletherapy for many different populations. ASD is unique in its presentation of symptomology, presenting some difficult cognitive, communication, and behavioral challenges. Additionally, many therapists report not feeling adequate to treat ASD (Solomon \& Chung, 2012). The Covid-19 pandemic has also highlighted the need for direction on using teletherapy for many different populations. Therefore, we present an adaption to Trauma-Focused Cognitive Behavioral Therapy to guide the therapist in using this model with children diagnosed with autism spectrum disorder and conducting therapy via teletherapy.

\section{Overview of Autism Spectrum Disorder \& Trauma}

Autism Spectrum Disorder (ASD) is a neurodevelopment disorder that affects 1 in 54 children (Maenner et al., 2020). According to the DSM 5, two characteristics define the diagnosis: Criteria A is deficits in social communication and social interaction, and Criteria $\mathrm{B}$ is restricted, repetitive patterns of behavior (APA, 2013). The diagnostic characteristics of ASD often cause children and adults to appear socially naïve and 
behave socially inappropriate (Kerns et al., 2015). Additionally, communication deficits are common with children diagnosed with ASD. $63 \%$ of children diagnosed with ASD meet the criteria to have a language disorder, making it difficult to have the language to communicate their struggles and experiences with others (Levy et al., 2010). These behaviors put individuals diagnosed with ASD in a vulnerable position to be victimized.

The relationship between ASD and trauma is a transactional relationship (Kerns et al., 2015). Meaning ASD may influence how trauma is experienced, and trauma may influence trauma-related symptoms or exacerbate ASD-related behaviors (Kerns et al., 2015). The effects of trauma experienced by individuals diagnosed with ASD are consistent with PTSD, meaning they may experience disturbances in arousal and reactivity (e.g., aggressive behavior, self-destructive behavior, concentration problems) and sleep difficulties (Peterson et al., 2019). Furthermore, children diagnosed with ASD who have a history of abuse have more intrusive thoughts, distressing memories, loss of interest, and lethargy (Brenner et al., 2017).

Caregivers and therapist often overlook the strengths of children with ASD. Identifying the strengths that an individual with ASD has will incorporate them into a strength-based approach to treatment (Cohen, 2012). There are many strengths attributed to individuals diagnosed with ASD, all these strengths may not be in each individual, but it is important to discover their strengths. Often individuals with ASD are visual learners and show typical or even advanced visualspatial processing details (Kumar, 2013). While autobiographical memory is often a challenge, rote memory is often a strength (Peterson et al., 2019). Many individuals with autism can tell you every exit on the freeway to their favorite destination. Others can recite lines from movies or music. As a therapist, it can become natural to focus on the limitations of the individual. Focusing on their strengths can change the course of treatment.

\section{Overview of TF-CBT}

While many treatment modalities show effectiveness when working with children and families exposed to trauma, Trauma-Focused Cognitive Behavioral Therapy (TF-CBT) is highly effective and has the most extensive evidence base of trauma-focused treatment modalities (Scheeringa et al., 2011). TF-CBT is a structured protocol that focuses on reducing trauma-related-symptoms and healing the trauma children, and their families have been exposed to (Ramirez de Arrellano et al., 2014). TF-CBT comprises trauma-based interventions with CBT principles, humanistic therapy, family therapy, and attachment theory. The core values of TF-CBT are summarized by the acronym CRAFT (Cohen et al., 2012):
Components-based, instilling knowledge, skills, and processes based on the client needs.

Respectful of individual, family, community, culture, and religious practices when working to understand the trauma's impact when supporting the child and family healing.

Adaptable therapists recognize the importance of finding flexible and creative ways to motivate the client while implementing the treatment protocol to diverse populations. Family-focused, therapists will make every effort to include family members in the treatment. This could include parents, caregivers, siblings, and grandparents.

Therapeutic relationship-centered therapists work to create an environment that allows parents and children to feel safe, accepted, and validated allowing clients to trust and feel confident in being able to share their traumatic experiences. Clients need to feel safe taking the risk necessary to share the most distressing fears, thoughts, and beliefs while being open to learning and utilizing new skills.

Key elements of the protocol include psychoeducation, coping skills, relaxation, affect regulation, cognitive coping, trauma narrative, cognitive processing, in-vivo exposure, and enhancing future safety. These elements are not only beneficial for the child but their families as well. A majority of the protocol should include the caregiver as this is a process in which the system is encouraged to learn about trauma and formulate strategies to reduce the role it plays in the system (Ramirez de Arellano et al., 2014). Many caregivers often hold space for guilt, as they feel they should have protected their child and were unable to. Fear of judgment and the fear that their child will be re-traumatized through treatment keeps caregivers from being engaged or wanting to be present in treatment. Therapists providing psychoeducation around the importance of caregiver participation and how their mental health impacts the reduction of their child's symptomology and well-being (Gopalan et al., 2010).

Current research has shown that TF-CBT over teletherapy is just as effective and beneficial as traditional in-person therapy in reducing PTSD symptoms and addressing several disorders that may develop after trauma is experienced (Stewart et al., 2017). A recent study examined PTSD symptoms in underserved trauma-exposed youth for the TF-CBT protocol and found a reduction in PTSD symptoms for all patients (Stewart et al., 2017). While each part of the TF-CBT protocol can be completed over a teletherapy format, one should still proceed with the same care and consideration they would when treating clients in person (Stewart et al., 2017).

\section{Overview of Teletherapy}

Teletherapy, or the practice of providing mental health services across distance with technology assistance, offers myriad 
benefits to individuals in need of therapeutic services (Turvey \& Myers, 2013). (Myers \& Turvey, 2013). Teletherapy offers unique solutions to individuals who live in mental health professional shortage areas, do not have access to a therapist specializing in their clinical concerns locally, and is both time and cost-effective (Stoll et al., 2020). These potential benefits align with the needs of families with a child diagnosed with ASD. These families are already at an increased burden in the number of services they receive, as individuals diagnosed with ASD may receive a range of treatment services, including applied behavioral analysis therapy, occupational therapy, speech therapy, and occupational therapy. Further, individuals diagnosed with ASD may struggle to find local services provided by professionals with training and expertise in working with autism (Johnson, 2019). Therefore, teletherapy provides a creative option to offer services to families who lack access to mental health services and are limited with time and ability to attend traditional same room therapy services.

As compared to in-person therapy, some research supports the idea that teletherapy reigns superior to in-person therapy due to technology allowing for more authentic reactions between children and their family members (Shealy et al., 2015). Teletherapy opens doors for deeper connections to be fostered since being in a safe and familiar setting could be more comfortable for them. (Stewart et al., 2017). Often, traumainformed treatment ignores the siblings within the system and other extended family members that may have been impacted. Teletherapy allows for these vital roles to be active in the system's healing process, which is not widely offered in traditional in-person sessions due to logistics (Gloff et al., 2015).

While there are many benefits to utilizing teletherapy services, it is important to note this medium's challenges. Online platforms bring the concern of privacy, confidentiality, security, and safety (Stoll et al., 2020). Thus, special consideration must be taken in locating secure teletherapy platforms and taking steps to protect the client's safety. Of critical importance, the use of teletherapy requires therapists to receive specialized training and education (Stoll et al., 2020). The problem is that many Commission on Accreditation for Marriage and Family Therapy Education (COAMFTE) accredited couple, marriage, and family therapy (CFT) programs report having no on site or external teletherapy clinical experiences (Pickens et al., 2019). Pickens et al., 2020.

Additionally, many programs do not offer a teletherapy curriculum in their program (Pickens et al., 2019 Pickens et al., 2020); therefore, today's practicing therapists may feel ill-prepared to deliver teletherapy services. An additional concern for therapists to consider is that the patients need to have compatible equipment in their homes for technology, and if they do have the right equipment, there may be technology disruptions to the session (Joseph et al., 2011). Presenting these challenges is not meant to persuade therapists to not use teletherapy services, but to stress the importance of pursuing training and education specific to teletherapy services to ensure that therapists who offer these services are due so legally, ethically, and with competency.

\section{Integration of TF-CBT for Autism \& Teletherapy}

As mentioned previously, children with autism are at greater risk of being exposed to traumatic events than their developing peers, and their experiences often go misunderstood or ignored (Hoover, 2015). Traumatic experiences that children with autism may face include but are not limited to emotional, physical, sexual, and neglectful abuse. All forms of abuse that qualify a child with autism to be a candidate for the TF-CBT protocol (Hoover, 2015). Due to the nature of co-occurring conditions being present in children with autism, it is important to seek prevention and intervention for these conditions (Kerns et al., 2017). Teletherapy allows for the evidencebased practice of TF-CBT interventions and preventions to be accessible to families and adolescents (Gloff et al., 2015).

When doing TF-CBT with children, careful considerations should be considered to provide the best treatment outcomes. Especially when working with children with autism, therapists should clearly understand how trauma manifests in children with autism to make appropriate adaptions of TF-CBT (Peterson et al., 2019). This paper aims to introduce ASDsensitive TF-CBT applications, allowing the therapist room for effective treatment and the management of the trauma symptoms present in children with ASD (Cohen et al., 2012). Due to the cognitive and language barriers in children with ASD, certain parts of the protocol may need to be adapted to fit the child's developmental level (Peterson et al., 2019).

When moving into portions of the protocol such as relaxation, cognitive coping/ processing, trauma narrative, and enhancing future safety, the therapist should consider their motivation behind doing these with children who have experienced trauma (Cohen et al., 2012). Frequently, therapists are inhibited by society's stigmas on children with autism and other developmental disabilities. It is important that when doing this protocol, one must be aware of any biases the therapist holds about the capabilities of ASD individuals (Cohen et al., 2012). This paper is not meant to teach all that is needed to work with ASD but how to adapt the protocol; if a therapist finds they have some bias they should seek more education. CBT offers great structure and skill-building that will provide effective treatment for children with ASD. Understanding the flexibility of TF-CBT allows for an unlimited world of interventions such as those from play therapy that could successfully adapt the TF-CBT protocol to children with autism and provide significant results in trauma symptoms (Cohen et al., 2012). 
Understanding each part of the protocol will help therapists to adapt. We will briefly discuss each part of the protocol and suggested adaptions. The protocol includes psychoeducation, coping skills, relaxation, affect regulation, cognitive coping, trauma narrative, cognitive processing, in-vivo exposure, and enhancing future safety.

\section{Assessment and Engagement}

One of the first steps in the TF-CBT protocol is assessment and engagement. Typically, assessment is done verbally or through the Trauma Symptom Checklist for Children (TSCC) and the Trauma Symptom Checklist for Young Children (TSCYC) (Wherry \& Dunlop, 2018). While these assessments will still be utilized, the Child and Adolescent Trauma Screen (CATS) also effectively assesses trauma symptoms in children and adolescents (Sachser et al., 2017). The CATS assessment provides an added layer to assessing for symptoms that the TSCC/TSCYC do not assess for such as specific behaviors, caregiver and child perceptions, and hypo/ hyperarousal levels. Having these unique areas allows therapists to gather more details of the experiences the system may have. It is also understood that this assessment is inclusive to children with autism (Sachser et al., 2017). A common characteristic of ASD is differences in social awareness and perception, which may impact the way events are interpreted. To counter this, the therapist should utilize as much of the identified client's system as possible to get the full story of the traumatic event (Peterson et al., 2019).

Doing these assessments verbally with the child will allow for the therapist to clarify the meaning of any of the questions. For instance, the TSCC asks questions that describe emotions, but many children diagnosed with ASD struggle to identify emotions. In this instance, the therapist can describe what it may feel like to be scared or sad; if the child still struggles with this, the therapist may have success using a scene in a movie that the child is familiar with to describe that emotion.

Therapists should plan to meet with the parents to assess for overlapping symptoms of trauma and ASD. Doing a thorough assessment can help a therapist identify what behaviors are ASD related and which behaviors may manifest trauma (Grosso, 2012). For example, the TSCC asks questions such as are you "wanting to yell and break things"; this may already have been part of the presentation of ASD for the child, so we would want to keep this in mind when doing assessments. This would include identifying what behaviors are recent since the time of the trauma and what behaviors have typically been a part of the presentation of autism behaviors.

Understanding what the behaviors relate to ASD or trauma the therapist will want to know how the behaviors manifest behaviorally. Understanding the behaviors will help a therapist feel confident in joining with and protecting the client. For instance, if a client engages in self-harm behaviors such as banging their head against the wall when overstimulated, the therapist needs to have a plan to inform parents or trusted caregivers to intervene.

A common characteristic of ASD is rigidity and routine. Thus, therapists should understand what their preferences are for routine and organization to alleviate the anxiety about a change in their life to better engage in sessions. As part of the assessment, the therapist should work with the parent to learn what patterns of rigidity are present and how the child handles change. A client's strong preference for rigidity can be used as an advantage in therapy as well. For instance, a therapist and client can come up with clear guidelines of therapy that are easy to follow, such as therapy sessions will last $50 \mathrm{~min}$, we will talk about these skills or topics today, or present an agenda at the beginning of each session. Preparing the child for what is to come may help alleviate anxiety; this could be presented in the visual format if they are visual learners (Peterson et al., 2019).

Establishing safety for future sessions is an important aspect of the assessment and engagement phase of therapy. It will be important to establish that if one of the parents is the offender to the client, then therapy needs to be done to feel safe sharing knowing that the parent cannot hear their sessions. This may mean having to do therapy when that parent is out of the house or finding a safe location that the parent cannot hear the sessions. If both parents are the offenders or the parents no longer have custody of the child, then work with the guardian or social worker assigned to the case to ensure the child has a safe environment to do therapy. Create a safety plan with the client that may include using a code word to let the therapist it is not safe to talk. Using room scans can help to establish a safe therapeutic environment on both sides of the call.

Teletherapy Adaptions While in traditional same room services, a family would be given these assessment measures in the waiting room to be completed before the start of the session, special consideration must be given to how assessments are completed with teletherapy. Therapists have a range of options to provide these measures to the family but must attend to confidentiality and privacy risks inherent in technology use. The therapist could plan on doing assessments by just asking the clients questions during a session; additionally, the therapist can utilize a confidential delivery platform to deliver the assessments.

In addition to the formal assessments used, therapists need to do some further assessments to prepare for teletherapy. When conducting therapy over technology, the therapist has less control of the environment, and special consideration must be given to how we prepare families for teletherapy and address unique considerations with the child's autism behaviors and triggers. While teletherapy provides families with the convenience of receiving services from their home, 
families are being asked to share the responsibility for developing a therapeutic environment in ways that are not required with traditional same room therapy services. Therapists should take time before the first session to inform families of ways to create a therapeutic space in their home, considering privacy issues, best practices for the use of technology, comfort, and unique issues related to their child with ASD.

\section{Psychoeducation}

The goal of psychoeducation is to normalize responses to trauma and educate on what the treatment protocol involves. It will be essential to use language that is developmentally appropriate for the child. Therapists should also inform parents that they will reach out to them before the teletherapy session to discuss the upcoming therapy session's specifics. Therapists should discuss with parents the goal for the session, materials required, time frame, and how the parents will be involved. The therapist can inform parents that these presession calls will be a routine part of treatment.

Children diagnosed with ASD often have short attention spans, so developing the materials for the session utilizing items from the child's interest may help to hold their attention. Along with visuals, therapists should slow down the process and even use a few words to present a simple message. Visuals include pictures, puppets, toys, and other materials that could be used to create stories (Grosso, 2012). Having a puppet on the screen may hold the child's attention more than looking at the therapist. Do not just rely on talk therapy but utilize activities and visual aids to help the child understand and learn. Grosso (2012) suggests using the book A Terrible Thing Happened (Holmes \& Mudlaff, 2000) as a helpful tool to teach about trauma. The book follows a raccoon who experienced a terrible thing and the story explores the racoon's symptoms of trauma through both words and pictures. While reading this book the therapist can stop and ask questions such as "Have you ever felt like that?", this can help connect the story to the experience of the child.

When providing psychoeducation, it is important to have an attitude of flexibility and patience. Children with ASD have cognitive limitations that can include poor comprehension and retention. Being flexible and patient allows the therapist to use repetition to teach the client. The use of repetition is a powerful tool in any therapeutic model, but it may be essential to help a child diagnosed with ASD retain knowledge (Grosso, 2012). Additionally, be flexible in the session's length as it may be helpful to shorten sessions if needed. Therapists should consistently check in with caregivers to assess optimal ways to join with and keep the client engaged in treatment (Grosso, 2012; Peterson et al., 2019).

Teletherapy Adaptions As therapists do not have access to the therapeutic space, they should contact parents in advance to discuss items that may be required for the session, such as arts and crafts or pillows, and guide the parents of the ways they will be involved in the session.

\section{Parenting}

One of the largest areas of the TF-CBT protocol is parenting. Parenting is the CBT belief that adolescents and children benefit the most from this form of treatment when there is parent support and participation (Wood et al., 2015). The ability to cater to the family system's protocol ensures that healing will permit future generations (Gloff et al., 2015). When it comes to caregiver participation, children with ASD may find it difficult to feel understood and comforted when faced with adverse experiences (Kerns et al., 2015). The reason being is that children with autism often lack the social support needed when healing or suffering from the effects of trauma (Hoover, 2015). Making it imperative that when doing teletherapy, the role of the caregiver and complete family system is taken into consideration to analyze how they will meet the child's needs and participate in the treatment process (Gloff et al., 2015). One benefit that therapists have in taking this systemic approach is the unique position of observing the family system in a more organic and natural setting. Observing these moments allows space for the therapist to understand what coping skills are used in the home and how they are or are not being practiced (Gloff et al., 2015). Doing so allows the therapist to understand which parts of the protocol have been achieved and which areas need to be addressed or re-addressed (Gloff et al., 2015).

\section{Relaxation}

Trauma affects the brain and affects the body (Van Der Kolk, 2014). The purpose of the relaxation protocol is to help the client connect and create awareness of the body. When introducing relaxation techniques, be mindful of the child's sensory needs. Some relaxation techniques include listening to music, dimming lights, or parents providing a weighted blanket (Peterson et al., 2019). The therapists can also teach the child grounding techniques such as balloon breathing. Balloon breathing involves the child putting their hands above their head, forming the shape of a balloon. As they breathe, they raise their hands higher in the air as if the balloon was feeling up with air. Then as they breathe out, their hands will lower as if the balloon was losing air. This can be a helpful and fun way to help the child practice breathing in and out slowly. The relaxation protocol is an excellent time to include a trusted parent or caregiver so the child can practice the technique at home and experience repetition between sessions. Parent mediated mindfulness interventions have shown to help reduce behaviors in a child with ASD (Hwang et al. 2015). Even though the therapist is not in the same room as the client, this 
does not take the ability to get up and move to demonstrate to the client the various techniques.

Teletherapy Adaptations Teletherapy allows for a unique opportunity to practice the relaxation techniques with the therapist's support where they may most often experience trauma response and where do the parents want to encourage the child to engage in relaxation techniques. If the technology allows for it, the session could be moved to the living room or the child's bedroom; this makes it possible to have the child be in the exact place that they want to practice the relaxation techniques. This should create confidence with the child and family that they are learning these techniques in the place in which they will use them. Utilization of what the client has at their home can be helpful to create relaxation.

\section{Affective}

The goal of affective modulation is to increase the capacity to identify a range of feelings, name the feelings, and identify appropriate expression. For children who experience typical development, not acknowledging emotions may lead to the child being vulnerable to depression due to not gaining access to the coping skills needed to regulate. However, too much focus on emotions may lead to a breach of that child's window of tolerance, leading them to have circular thoughts or worry (Rieffe et al., 2015). To understand the realms of the child's window of tolerance, a subjective unit of distress scale (SUDs) is utilized (Kivimba \& O'Reilly, 2017). This scale is a simple scale that typically ranges from 0 to 10 to identify the intensity of emotions (Kivimba \& O'Reilly, 2017). However, this scale can also include different zones to identify feelings if the child is having difficulty utilizing a 1-10 scale. For example, children may identify a calm zone, stressed zone, and overwhelmed zone to place a range of emotions rather than have to identify ten emotions. Putting this into practice, a therapist can pull out a whiteboard and have a child pull out a piece of paper at home. Using the whiteboard, the therapist can show the child how to draw out these zones while the child draws their paper zones. The therapist then will help the child outline behaviors that they see in each of these zones. Many children with autism struggle to identify emotions, so identifying the zones and focusing on behaviors can begin to open the conversation to discuss emotions in each zone.

Children with autism experience these two aspects of emotional awareness in a different way. Children with autism have a more fragmented understanding of their emotions and emotional experience. They are more likely to observe the different emotional experiences that allow them to adapt and quickly mimic their environment. Studies have shown that children with autism have fewer coping strategies due to this. Children with autism have a difficult time understanding their emotions and psychological and contextual factors that impact emotional awareness (Peterson et al., 2019). Individuals with ASD often rely on repetitive behaviors to regulate the intense emotions they may be feeling. This could be due to the difficulties of utilizing other coping skills such as altering thoughts or finding ways to disrupt distressing situations. This could impact how and when a child with ASD experiences trauma (Kerns et al., 2015). That is why it is important when implementing the affect regulation of the protocol to understand the child's experience and allow space to develop these coping strategies (Rieffe et al., 2011).

More than with other populations, therapists may need to slow sessions down here and utilize repetition to help the client with autism to learn to recognize emotions. The use of visuals can help the client slow down to identify and monitor emotions; visuals such as a stop sign can be used to remind the client to stop and monitor. Another tactic that the therapist may find useful is rule-based teaching, drawing on the strength of routine, rigidity, and rules that an individual with ASD often displays. For example, rule-based teaching will say: "When you feel sad, use this skill;", "When you have this thought, do this...". Taking time in this section will prepare the client for cognitive coping and some of the more distressing phases, such as in vivo exposure (Peterson et al., 2019).

Teletherapy Adaptions A therapist can have their copy of the visual while providing a client with a copy via email or sending a package to the houses with the therapy supplies.

\section{Cognitive Coping}

Children with ASD typically struggle with abstract or critical thinking, making this one of the more difficult protocol elements. To teach the child about thought it is helpful to reframe how thinking is described; thinking can be described as "talking to ourselves" asking the client "What do you say to yourself when you make a mistake? (Cohen, 2012, p. 164). This section's main purpose is to teach cognitive coping skills, acknowledge and share internal dialogue, and understand the relationship between thoughts, feelings, and behaviors (known as the CBT triangle). Simply put, the goal is to help the client connect the talking they do to themselves and connect to feelings and emotions. As the cognitive level of every child differs, the therapist should tailor towards their individual needs.

Cohen (2012) provides some interesting ideas on how to overcome the challenges of teaching cognitive coping. One interesting method is to play "baseball" with the client. This can be accomplished virtually, possibly with parents or caregivers at home, creating a triangle in the child's room. The triangle represents the $\mathrm{CBT}$ triangle. First, the client goes to the thought corner, give the client a scenario followed by questions such as, "What are you thinking? What are you 
saying to yourself?" Then move the client to the feeling corner and ask, "How are you feeling?" And then jump to the behavior corner where the client is asked, "What do you do?" Then process the thoughts, feelings, and behaviors the client had within the scenario. Continue using repetition and moving slowly to be sure the client can make the connections.

Teletherapy Adaptions As we are doing this virtually, make sure that the client can still hear the therapist even when stepping away from the computer.

\section{Trauma Narrative}

In preparation for the trauma narrative, having a positive reinforcement pattern and constant praise can help the child associate positive feelings as they apply their coping skills and soothe themselves through the experience (Grosso, 2012). Often parents struggle to utilize positive reinforcement, so this is a powerful opportunity for the therapist to demonstrate how to praise the child.

A trauma narrative is a powerful tool used to help the client recount the traumatic experience without pairing the experience with negative thoughts, triggers, and emotions. The therapist will help the client process the story while utilizing the coping and relaxation skills that have been taught previously. Children with ASD have a proneness toward anxiety (Grosso, 2012), which could trigger self-injurious behaviors. Knowing this, the therapist needs to have a plan with the child and the parent to keep the child safe from their behaviors as the therapist will not be in the same room as the client. Part of this plan could be preparing the child with social stories the therapist has written that describe a child who is anxious and utilizes their coping skills. The use of repetition with the social stories will help prepare the child for what may come.

Teletherapy Adaption The author has utilized social stories via teletherapy by sharing the therapist screen with a word document. With the help of the client, a story is written; by sharing the screen the client can see the story being typed out while the therapist reads the story out loud. Then share the story with the client via a confidential platform. The client can print the story out and illustrate the story. Remind parents before the session to have the supplies on hand for the child to tell their story.

\section{Cognitive Processing}

Like other children who experience trauma, a child diagnosed with ASD may experience cognitive distortions such as blame, guilt, or fear. The challenge with processing distortions is that children with ASD often have cognitive or processing delays, causing them to have a lower threshold for negative experiences and abstract thinking (Grosso, 2012). Returning to the story written in the trauma narrative can help the therapist, and the client creates a visual storyboard. This visual storyboard can be drawings the client creates about the trauma narrative where the client can identify how the character in the story may be feeling. This is the time for the therapist to explore the trauma narrative and process further what meaning the client creates about the experience. For instance, the client feels "bad" that people got in trouble, indicating guilt and blame the client has internalized.

\section{In-Vivo Exposure}

The goal of in-vivo exposure is to help the child to decrease the avoidance of negative emotions. To prepare a child with ASD for exposure, it will be important to create a step by step plan to introduce smaller increments of escalating stimuli (Grosso, 2012; Peterson et al., 2019). With in-vivo exposure, we want to reduce avoidance behaviors that interfere with daily functioning. To complete this, the therapist can create a fear ladder with the client, identifying different levels of fear and triggers for each fear. This can be an important time to utilize a parent or caregiver as children with ASD often do not have self-awareness to recognize these avoidant behaviors.

When children with ASD are exposed to change or tense situations, they often return to their rote behaviors (e.g., body rocking, hand flapping). When the therapist notices this behavior, they should intervene so it does not become a habit. Intervening with rote behaviors can be intervened by returning to the relaxation techniques established earlier in therapy. For instance, they can practice some balloon breathing, put on a weighted blanket, or listen to some calming music. Therapist should return to the SUD's scale the client during the affect stage to help the client recognize the level of stress they are currently experiencing as levels of exposure are increased. Parents should be involved in this process to provide support, consistency, and reinforcement (Grosso, 2012). The therapist may schedule a separate video session with the parents to create a plan that they are comfortable with and then coach them in a session with the child on implementing the plan.

\section{Enhancing Future Safety}

Children with ASD often struggle to understand social cues and socially appropriate behaviors (Kerns et al., 2015). To create safety for the child, the therapist will need to help the child distinguish between acceptable and unacceptable. Be explicit in helping the client understand who it is safe to share thoughts and experiences with and who is not safe. Outline specific behaviors that may be inappropriate such as sitting on someone's lap or hugging others may be unsafe. This could be when the therapist could lead the client and family members through role plays outline safe versus unsafe. 
Teletherapy Adaption Utilizing teletherapy if the child is at home and it is safe and confidential, the therapists can have the client and family role-play the scenarios in different parts of the house to experience the role play in various scenarios situations. As with the other tasks, this may require repetition (Peterson et al., 2019).

\section{Additional Clinical Recommendations}

Whenever possible, therapists should involve a trusted caregiver or parent, using parents or caregivers can help communication between parents and children while increasing their comfort level in talking about trauma (Grosso, 2012). Children with ASD often have difficulty engaging in conversation with others, conjoint sessions with parents will provide opportunities to help these children learn this important skill. Learning to communicate could enhance the child's safety as they learn to express their emotions or speak out against potential harm.

Children with ASD are comfortable with routine and struggle with unexpected change, when transitioning from a preferred activity to a less preferred activity, they give the child advanced warnings. To help prevent resistance or burnout in therapy, it may be a good idea for the therapist to work with the parents to create a sensory box used only in sessions as reinforcement. This could look like toys the client enjoys, play doh, a weighted blanket, or calming music. Depending on the client's control capabilities, it may be needed to have a parent control the box and give the child access to the box at either planned, earned, or needed breaks. Using the parents, we can work to prevent overstimulation to sensory sensitivities as well.

Overstimulation can be a struggle for children with ASD. Therapists will want to meet with parents ahead of time to prepare the room that therapy will occur in for both the client and the therapist. Parents may need to make changes in the home therapy environment to reduce distractions and provide a safe place if they have any self-injurious behaviors. Additionally, the therapist will want to pay attention to what the child may see on camera to ensure they are not overstimulating or distracting. As part of the assessment process, it will be the therapist's advantage to learn what sensory sensitivities the client may have, so the therapist should be sure not to have bright colors, a fan spinning, or distracting toys in the background.

Using concrete, firm language is important for the child to have a sure understanding of what is expected of them such as no hitting or use your words (Peterson et al., 2019). The therapist will want to discuss with parents what language they use to stop behaviors or engage the client, this will allow for consistency in reinforcement or punishment that the child experiences at home.
The final recommendation is to remember to slow down and use fewer words. Children with ASD take longer to process so the therapist should adapt to their cognitive level. Using other communication strategies such as first, then contingencies will increase compliance in session (e.g., first we will practice our coping skills, then you can have a break with your sensory bin). Therapists should be aware of how even brief periods of silence may be perceived when communicating through technology. Clients, particularly the parents may worry that periods of silence indicate a loss in connection or a frozen screen and therapists should consider how slowing things down or allowing for silence for the child to process could raise anxiety over technology-related issues. Therapists can address this by limiting the use of silence in sessions when needed but especially by informing the clients ahead of time using silence as a technique or practice with ASD clients to allow them to process. Other communication strategies that will increase success include repetition and practice to ensure the child comprehends the material, it is okay to repeat material over multiple sessions to help the child learn. The repetition utilizes multiple learning methods at once, such as using both visual and verbal to present new information (Peterson et al., 2019).

\section{Future Research}

We recognize that this paper is theoretical; we have confidence that our suggestions for ASD and telehealth will help a therapist in their work; we also recognize the need for evidence-based treatment with this population. Despite the rising prevalence of ASD and the vulnerable position that children with ASD experience, there is not enough research to understand the presentation of PTSD symptoms (Peterson et al., 2019). There is a strong need for empirical research on telehealth's efficacy, especially among ASD families.

\section{Limitations}

While TF-CBT is effective over teletherapy (Stewart et al., 2017) and we believe it will be effective in working with children with autism (Peterson et al., 2019), it is important to understand the limitations that technology may cause for the treatment process. Technical difficulties are reported to be the largest disruption to the treatment process. For those with slow-speed internet connection or limited access to technology it could be difficult to proceed with consistent treatment. Aside from technology, children with autism often manifest their emotions and trauma symptoms in ways that may look different than their developing peers. These symptoms may be missed or overlooked by peers, therapists, family, and schoolteachers (Hoover, 2015). While these limitations may exist, there are training implications for these limitations to be minimized. 


\section{Training Implications}

As therapists take the time to learn and understand the protocol's adaptions, they will find more success and develop confidence in working with this population. Many platforms will train therapists to use TF-CBT to feel confident in implementing and adapting the protocol. For example, there is online training available through the Medical University of South Carolina (MUSC, 2017). Additionally, the therapist should take the time to become familiar with the technology they plan on using and have a developed plan to solve technology problems if they occur.

As was discussed earlier, a therapist should examine any biases or misconceptions about ASD they have. Many therapists do not feel confident working with ASD as they may have received little training on ASD in academic training. The Asperger/Autism Network (AANE, 2021) offers mental health clinicians resources to gain more competence in this area. The International Board of Credentialing and Continuing Education Standard (IBCCES, 2021) offers a Certified Autism Specialist certificate to further one's credentials with ASD clients.

Trauma-Focused CBT has strong empirical evidence of its effectiveness, and with the proper. For many families with children who have ASD, it can be difficult for them to have the resources to get out of the house (Romney et al., 2021); being able to deliver services in the home can ease the stresses of the family.

\section{Declarations}

Research Involving Human and Animal Participants This manuscript did not involve any human or animal participants. It is a clinical paper. Any discussion of research findings refers to previously published research.

Conflict of Interest There were no potential conflicts of interest.

\section{References}

American Psychiatric Association. (2013). Diagnostic and statistical manual of mental disorders (5th ed.). https://doi.org/10.1176/appi. books. 9780890425596

The Asperger / Autism Network (AANE). (2021, March 8). Asperger Syndrome (Asperger's) \& Autism / ASD. https://www.aane.org/.

International Board of Credentialing and Continuing Education Standard (IBCCES) (2021, February 12). Autism \& Cognitive Disorder Certification, Training \& CEUs. IBCCES. https://ibcces.org/.

Cohen, J., Mannarino, A., \& Deblinger, E. (2012). Trauma-focused CBT for children and adolescents: Treatment applications.

De Arellano, M. A., Lyman, D. R., Jobe-Shields, L., George, P., Dougherty, R. H., Daniels, A. S., Ghose, S. S., Huang, L., \& Delphin-Rittmon, M. E. (2014). Trauma-focused cognitive-behavioral therapy for children and adolescents: Assessing the evidence.
Psychiatric services (Washington, D.C.), 65(5), 591-602. https:// doi.org/10.1176/appi.ps.201300255.

Gloff, N., LeNoue, S., Novins, D., \& Myers, K. (2015). Telemental health for children and adolescents. International Review of Psychiatry (Abingdon, England), 27(6), 513-524.

Gopalan, G., Goldstein, L., Klingenstein, K., Sicher, C., Blake, C., \& Mckay, M. (2010). Engaging families into child mental health treatment: Updates and special considerations. Journal of the Canadian Academy of Child and Adolescent Psychiatry = Journal De L'Academie Canadienne De Psychiatrie De L'enfant Et De L'adolescent, 19(3), 182-196.

Grosso, C. A. (2012). Children with developmental disabilities. Treatment Applications: Trauma-Focused CBT for Children and Adolescents.

Holmes, M. M., \& Mudlaff, S. J. (2000). A terrible thing happened. Washington, DC: Magination Press.

Hoover, D. (2015). The effects of psychological trauma on children with autism spectrum disorders: A research review. Review Journal of Autism and Developmental Disorders, 2(3), 287-299.

Hwang, Y. S., Kearney, P., Klieve, H., Lang, W., \& Roberts, J. (2015). Cultivating mind: Mindfulness interventions for children with autism spectrum disorder and problem behaviors, and their mothers. Journal of Child and Family Studies, 24, 2093-3106. https://doi. org/10.1007/s10826-015-0114-x.

Johnson, J. (2019). Family therapy. In R. Rieske (Ed.), Handbook of interdisciplinary treatments for autism Spectrum disorder. Cham: Autism and Child Psychopathology Series. Springer.

Joseph, V., West, R., Shickle, D., Keen, J., \& Clamp, S. (2011). Key challenges in the development and implementation of telehealth projects. Journal of Telemedicine and Telecare, 17(2), 71-77.

Kerns, C., Newschaffer, M., \& Berkowitz, C. (2015). Traumatic childhood events and autism spectrum disorder. Journal of Autism and Developmental Disorders, 45(11), 3475-3486.

Kerns, C., Newschaffer, M., Berkowitz, C., Lee, S., \& Brian, K. (2017). Brief report: Examining the association of autism and adverse childhood experiences in the national survey of children's health: The important role of income and co-occurring mental health conditions. Journal of Autism and Developmental Disorders, 47(7), 22752281.

Kiyimba, N., \& O'Reilly, M. (2017). The clinical use of subjective units of distress scales (suds) in child mental health assessments: A thematic evaluation. Journal of Mental Health, 1-6.

Levy, S. E., Giarelli, E., Lee, L. C., Schieve, L. A., Kirby, R. S., Cunniff, C., Nicholas, J., Reaven, J., \& Rice, C. E. (2010). Autism spectrum disorder and cooccurring developmental, psychiatric, and medical conditions among children in multiple populations of the United States. Journal of Developmental and Behavioral Pediatrics, 31, 267-275.

Maenner M.J, Shaw K.A., Baio J., et al. (2020) Prevalence of autism spectrum disorder among children aged 8 years. Autism and Developmental Disabilities Monitoring Network, 11 sites, United States, 2016. MMWR Surveill Summ; 69(no. SS-4):1-12. https:// doi.org/10.15585/mmwr.ss6904alexternalicon.

Myers, K., \& Turvey, C. L. (Eds.). (2013). Telemental health: Clinical, technical, and administrative foundations for evidence-based practice. Elsevier.

Peterson, J. L., Earl, R. K., Fox, E. A., Ma, R., Haidar, G., Pepper, M., Berliner, L., Wallace, A. S., \& Bernier, R. A. (2019). Trauma and autism spectrum disorder: Review, proposed treatment adaptations and future directions. Journal of Child \& Adolescent Trauma, 12(4), 529-547. https://doi.org/10.1007/s40653-019-00253-5.

Pickens, J. C., Morris, N., \& Johnson, D. J. (2020). The digital divide: Couple and family therapy programs' integration of teletherapy training and education. Journal of Marital and Family Therapy, 46(2), 186-200. https://doi.org/10.1111/jmft.12417. 
Romney, J., Austin, K., Fife, S. T., Sander, D., \& Snyder, H. (2021). Stress experienced and meaning-making of couples with children with autism spectrum disorder: A phenomenological study. The American Journal of Family Therapy, 49(1), 37-56. https://doi. org/10.1080/01926187.2020.1813654.

Rieffe, C., Oosterveld, P., Terwogt, M., Mootz, S., Van Leeuwen, E., \& Stockmann, L. (2011). Emotion regulation and internalizing symptoms in children with autism spectrum disorders. Autism, 15(6), 655-670.

Sachser, C., Berliner, L., Holt, T., Jensen, T., Jungbluth, N., Risch, E., et al. (2017). International development and psychometric properties of the child and adolescent trauma screen (CATS). Journal of Affective Disorders, 210, 189-195.

Scheeringa, M., Weems, C., Cohen, J., Amaya-Jackson, L., \& Guthrie, D. (2011). Trauma-focused cognitive-behavioral therapy for posttraumatic stress disorder in three-through six year-old children: A randomized clinical trial. Journal of Child Psychology and Psychiatry, and Allied Disciplines, 52(8), 853-860.

Shealy, K., Davidson, T., Jones, A., Lopez, C., \& De Arellano, M. (2015). Delivering an evidence-based mental health treatment to underserved populations using telemedicine: The case of a traumaaffected adolescent in a rural setting. Cognitive and Behavioral Practice, 22(3), 331-344.

Solomon, A. H., \& Chung, B. (2012). Understanding autism: How family therapists can support parents of children with autism spectrum disorders. Family Process, 51(2), 250-264. https://doi.org/10.1111/j. 1545-5300.2012.01399.x.

Stewart, R., Orengo-Aguayo, R., Cohen, J., Mannarino, A., De Arellano, M., Baggett, K., \& Self-Brown, S. (2017). A pilot study of trauma- focused cognitive-behavioral therapy delivered via telehealth technology. Child Maltreatment, 22(4), 324-333.

Stoll, J., Müller, J. A., \& Trachsel, M. (2020). Ethical issues in online psychotherapy: A narrative review. Frontiers in Psychiatry, 10(993). https://doi.org/10.3389/fpsyt.2019.00993.

Storch, E., Sulkowski, A., Nadeau, M., Lewin, L., Arnold, J., Mutch, A., et al. (2013). The phenomenology and clinical correlates of suicidal thoughts and behaviors in youth with autism Spectrum disorders. Journal of Autism and Developmental Disorders, 43(10), 2450 2459.

Medical University of South Carolina (MUSC) (2017). TF-CBT Web 2.0. https://fcbt2.musc.edu/

Van der Kolk, B. (2014). The body keeps the score: Brain, mind, and body in the healing of trauma. Viking.

Wood, J., Ehrenreich-May, J., Alessandri, M., Fujii, C., Renno, P., Laugeson, E., et al. (2015). Cognitive behavioral therapy for early adolescents with autism Spectrum disorders and clinical anxiety: A randomized, controlled trial. Behavior Therapy, 46(1), 7-19.

Publisher's Note Springer Nature remains neutral with regard to jurisdictional claims in published maps and institutional affiliations.

Justin Romney M.S. is a doctoral student in the Couple, Marriage, and Family Therapy Program at Texas Tech University. Miranda Garcia, M.S. is a graduate of Couple, Marriage, and Family Therapy Program at Texas Tech University and is employed at the Children's Advocacy Center of the South Plains 